\title{
Pliometría y velocidad en jóvenes tenistas*
}

\author{
Jorge Mauricio Celis Moreno* \\ Esteban Sabogal Alarcón $n^{* * *}$
}

Recibido: noviembre 12 de 2016 • Evaluado: diciembre 5 de 2016

Aceptado: diciembre 15 de 2016

Resumen

Este estudio investigó los efectos de un programa de entrenamiento pliométrico y de velocidad de ocho semanas en jóvenes tenistas $(n=12)$ : seis hombres (edad: $13.87 \pm 0.94$ años, altura: $161.17 \pm 6.5 \mathrm{~cm}$, peso: $45.82 \pm 5.57$ $\mathrm{kg}$ ) y seis mujeres (edad: $14.83 \pm 1.38$ años, altura: $154.22 \pm 7.06 \mathrm{~cm}$, peso: $49.45 \pm 9.15 \mathrm{~kg}$ ). Los protocolos de salto horizontal, velocidad en $20 \mathrm{~m} \mathrm{y}$ agilidad 10 x 5 m fueron evaluados antes, durante y al finalizar el programa. Se determinó el estado de maduración y se elaboró el perfil antropométrico. Los resultados muestran que después del programa de entrenamiento todas las pruebas mejoraron, principalmente el salto horizontal en las mujeres $(\mathrm{p} \leq 0.05)$, velocidad en $20 \mathrm{~m}$ y agilidad $10 \times 5 \mathrm{~m}$ en hombres $(\mathrm{p} \leq 0.05)$. En conclusión, los tenistas mejoraron en los protocolos después de participar en el programa de entrenamiento, siendo un hallazgo importante para el desempeño en campo.

Palabras clave: pliometría, velocidad, jóvenes, entrenamiento, tenis.

"Artículo de investigación científica original, inició en agosto de 2016 y finalizó en noviembre de 2016 .

** Profesional en cultura física, deporte y recreación, magíster en entrenamiento deportivo para niños y jóvenes. Ex preparador físico de la Liga de Tenis de Bogotá. Correo electrónico: maito419@hotmail.com

*** Profesional en cultura física, deporte y recreación. Ex practicante de preparación física de la Liga de Tenis de Bogotá. Correo electrónico: esteban.sabogal@usantotomas.edu.co 


\section{Plyometric and speed in youth tennis players}

\section{Abstract}

This study examined the effects of a 8 week plyometric and speed training program in youth tennis players $n=12$; Six boys (age: $13,87 \pm 0,94$ years, body height: $161,17 \pm 6,65 \mathrm{cms}$, body mass: $45,82 \pm 5,57 \mathrm{kgs}$ ) and six girls (age: $14,83 \pm 1,38$ years, body height: $154,22 \pm 7,06 \mathrm{cms}$, body mass: $49,45 \pm 9,15 \mathrm{kgs})$. Pre , during and post test included horizontal jump, $20 \mathrm{mts}$ and $10 \times 5 \mathrm{mts}$ agility was assessment. Somatic maturity status was determined and anthropometric profile was described. The results showed that, after training program each test improved, mainly horizontal jump in girls $\mathrm{p} \leq 0.05,20 \mathrm{mts}$ and $10 \mathrm{x} 5 \mathrm{mts}$ agility in boys $\mathrm{p} \leq 0.05$. In conclusion, youth tennis players after of the training program improved on specific protocols and this is important to the performance on the court.

Keywords: Plyometric, speed, youth, training, tennis. 


\section{Introducción}

$\mathrm{Al}$ igual que otros deportes el tenis de campo requiere de diferentes aspectos físicos, psicológicos y técnico-tácticos (Kovacs, 2007). Desde la parte física, algunas de las características más importantes son los esfuerzos intensos, como aceleraciones, desaceleraciones, cambios de dirección y movimientos explosivos, los cuales tienen gran predominancia; estos esfuerzos intensos vienen acompañados de tiempos de baja intensidad, como el descanso entre cada punto (20 s) y en cambios de lado (90-120 s) (Fernández-Fernández, Kinner y Ferrauti, 2010; Kovacs, 2007). Por lo anterior, el tenis de campo se convierte en un deporte para el cual el entrenamiento específico de la potencia merece gran atención, con el fin de mejorar los movimientos explosivos que lo identifican (Fernández-Fernández, Saez de Villarreal, Sanz Rivas y Moya, 2016; Berdejo y González, 2009). De acuerdo con Torres-Luque y colaboradores (2011), las características de competición en jóvenes tenistas son $105 \pm 20$ min de duración de juego, 31.50 min de tiempo real de juego, y $73.5 \pm 8.50$ min de tiempo de descanso, lo cual demuestra que la estructura temporal es muy parecida a la de jugadores profesionales, a pesar de que existen diferencias en la condición física y en el desarrollo de la técnica, factores que determinan tales cualidades (Kovacs, 2006).

La fuerza explosiva es una capacidad asociada a la velocidad de un movimiento (Chu, 2003) y la agilidad está relacionada con la habilidad de cambiar de dirección rápidamente (Fernández-Fernández et al., 2016). Estas características tienen gran influencia en el campo de juego, ya que un jugador de tenis en promedio recorre en la cancha de 8 a 15 metros en el transcurso de un punto y cambia de dirección frecuentemente (Fernández-Fernández et al., 2010; Kovacs, 2006). Por lo tanto, el entrenamiento pliométrico toma importancia, debido a su componente explosivo, que ayuda a los tenistas a generar una transferencia de fuerza y responde a su necesidad de dinamismo al hacer movimientos multidireccionales (Fernández-Fernández et al., 2016). La efectividad del programa de entrenamiento pliométrico y dicha transferencia de fuerza dependen de la optimización de las cargas, adecuando el manejo de intensidad, volumen y frecuencia de los ejercicios (Sarabia et al., 2015); también se recomienda que estos programas se realicen entre 2 y 3 veces por semana, y se combinen con entrenamiento de otras capacidades, como parte del programa (Jastrzebski et al., 2014), para no tener sobrecargas en los tenistas y permitir tiempos óptimos de recuperación. 
Existen diferentes estudios que relacionan fuerza explosiva, agilidad y otras capacidades (Mateescu, 2013; Váczi et al., 2013; Alptekin, Kilik y Mavis, 2013; Mohd, Syed y Shamsul, 2014; Jastrzebski et al., 2014), algunos de ellos específicamente en el tenis (Fernández-Fernández et al., 2016; Sarabia et al., 2015). Sin embargo, pocos de ellos hacen control del estado de maduración de la población en edades adolescentes, a pesar de que la relevancia de esta variable ha sido bien sustentada en diferentes investigaciones (Malina, Bouchard y Bar, 2004). El objetivo de este estudio fue investigar los efectos de un programa de entrenamiento pliométrico y de velocidad con una duración de ocho semanas sobre tres protocolos estandarizados de velocidad $(20 \mathrm{~m})$, agilidad $(10 \times 5 \mathrm{~m})$ y fuerza explosiva en miembros inferiores (salto horizontal) en tenistas de élite.

\section{Metodología}

El grupo de muestra fue de 12 jugadores de tenis, conformado por 6 hombres (13.17-15.69 años) y 6 mujeres (12.81-16.38 años). Los participantes pertenecen al grupo élite de la Liga de Tenis de Bogotá, cada jugador tiene ranking de la Federación Colombiana de Tenis en sus respectivas categorías: 12-14 años y 14-16 años. Todos entrenan 15 horas semanales y tienen más de 2 años de experiencia en la práctica de este deporte.

\section{Procedimiento}

La participación de los jugadores en este proyecto fue voluntaria. La Liga de Tenis de Bogotá fue informada sobre este proceso experimental. Además, los investigadores obtuvieron la firma del consentimiento informado para padres o para la persona responsable de cada jugador.

Los jugadores fueron evaluados antropométricamente antes de comenzar el programa y fueron evaluados físicamente en tres momentos diferentes: antes de iniciar (T1), en la mitad del proceso (T2) y al finalizar el programa (T3). Los tenistas participaron en el programa durante ocho semanas, en el cual desarrollaron su entrenamiento de tenis como de costumbre. El programa se llevó a cabo en la hora de preparación física, que normalmente tiene lugar cada día después del entrenamiento técnico-táctico en campo, tres veces por semana (lunes, la mitad de las sesiones de martes y el miércoles). Se 
destinó el jueves para juegos recreativos y el viernes, para sesiones de fortalecimiento y prevención de lesiones. En la figura 1 se puede observar el diseño y la organización del programa planteado.

Figura 1. Diseño del programa de entrenamiento

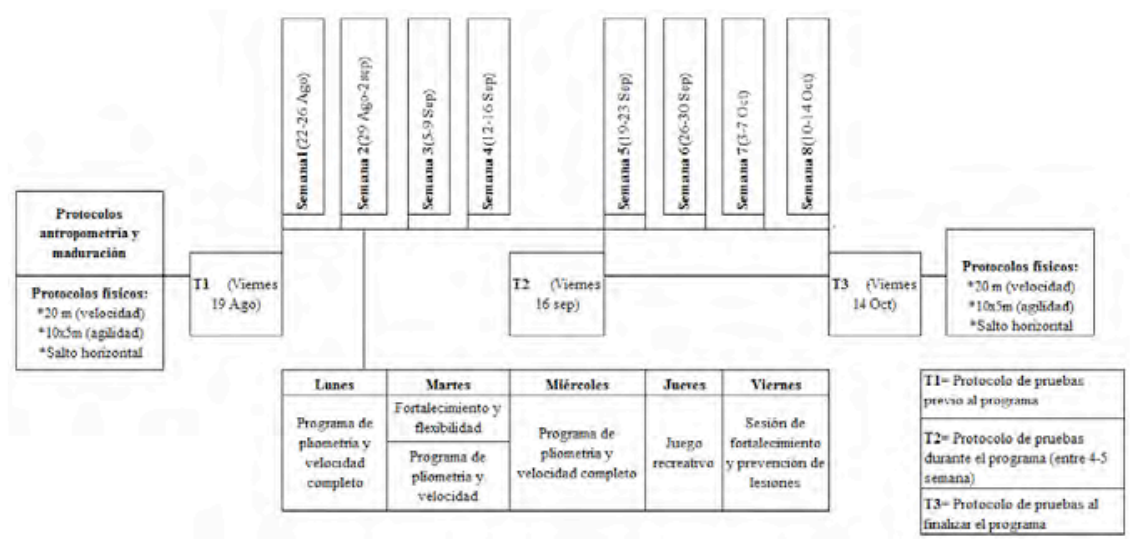

Fuente: elaboración propia.

Las prioridades de este programa fueron ejercicios pliométricos de multisaltos, ejercicios de velocidad de 15 y $20 \mathrm{~m}$, salto de lazo, y la combinación de salto más carrera de velocidad de $20 \mathrm{~m}$. La descripción del trabajo realizado durante las ocho semanas del programa de entrenamiento se puede observar en la tabla 1. 
Tabla 1. Descripción del trabajo realizado

\begin{tabular}{|c|c|c|c|c|c|}
\hline \multirow{2}{*}{ Salto lazo } & Repeticiones & \multicolumn{4}{|c|}{ Semana } \\
\hline & & $1-2$ & $3-4$ & $5-6$ & $7-8$ \\
\hline & 300 & $\sqrt{ }$ & & & \\
\hline & 500 & & $\sqrt{ }$ & & $\sqrt{ }$ \\
\hline & 700 & & & $\sqrt{ }$ & \\
\hline Velocidad $15 \mathrm{~m}$ (sin carga) & 10 & & $\sqrt{ }$ & & \\
\hline & 15 & & & & \\
\hline Velocidad 20 m (sin carga) & 10 & $\sqrt{ }$ & & $\sqrt{ }$ & \\
\hline Velocidad 20 m (con carga: balón 2 kg) & 10 & $\sqrt{ }$ & & $\sqrt{ }$ & $\sqrt{ }$ \\
\hline Multisaltos (x 8) de 15-20 cm & 10 & $\sqrt{ }$ & & & $\sqrt{ }$ \\
\hline Multisaltos (x 8) de 20-30 cm & 15 & & $\sqrt{ }$ & $\sqrt{ }$ & \\
\hline Combinación 3 saltos - velocidad 20 m & 10 & & $\sqrt{ }$ & & $\sqrt{ }$ \\
\hline
\end{tabular}

$*(\mathbf{x} 8)$ cantidad de saltos por cada repetición.

Fuente: elaboración propia.

\section{Antropometría y estado de maduración}

La fecha de nacimiento, altura, altura sedente y peso corporal fueron evaluados, al igual que siete pliegues cutáneos (tríceps, subescapular, bíceps, suprailiaco, abdominal, muslo y pantorrilla). El método de evaluación para porcentaje graso usado se basó en la propuesta de Slaughter (1988) para niños y jóvenes. Todas las evaluaciones fueron realizadas por el mismo antropometrista. La edad cronológica fue determinada por la fecha de nacimiento y la fecha en la cual ellos fueron evaluados. Fue evaluada la proyección de estatura adulta con base en el protocolo propuesto por Kamis y Roche (1995); como medio de control del estado de maduración somática se usó el maturity-offset propuesto por Mirwald et al. (2002), y finalmente se estableció su nivel de maduración —atrasado, normo-maduro y adelantado-, según lo planteado por Malina et al. (2004).

\section{Evaluación física}

Se utilizaron tres protocolos de evaluación física: salto horizontal sin impulso, las pruebas de velocidad de 20 metros lanzados y de agilidad de 10 x 5 metros. Para evaluar el salto horizontal, se tuvo en cuenta la normatividad 
propuesta por EUROFIT. El participante se ubicó detrás de la marcación donde comenzó la extensión de la cinta métrica. Luego, realizó dos saltos de calentamiento y un minuto después de que se le explicara el protocolo, hizo tres intentos, con un descanso entre 30 y 45 segundos entre cada intento. El evaluador estuvo cerca al punto de caída y marcó señalando con el dedo el talón del pie más atrasado en la caída. Adicionalmente, se registró la distancia en centímetros.

Para la prueba de velocidad de 20 metros lanzados, el participante se ubicó en la zona de comienzo y dos evaluadores, en la línea de llegada de la distancia recorrida. Cada participante podía hacer dos intentos. El estímulo de partida fue un pitazo. Ambos evaluadores registraron el tiempo y el tiempo utilizado fue la media del mejor de los dos intentos. El tiempo se registró en segundos con dos decimales.

Finalmente, en la prueba de agilidad de 10 x 5 metros, el participante recorrió una distancia de 5 metros, 10 veces consecutivas sin parar. El protocolo se aplicó con la normatividad propuesta por EUROFIT, el tiempo fue registrado por dos evaluadores cada uno con su cronómetro, quienes se ubicaron en la línea de llegada. La prueba se realizó en un intento y el tiempo utilizado fue la media del registro de cada evaluador. El tiempo se registró en segundos con 2 decimales.

Para todos los protocolos, se usaron los siguientes materiales: adipómetro Caliper Slim, cinta métrica antropométrica Gulick, tallímetro de pared Kramer 2104, báscula de peso corporal Beurer BF66, cronómetros Casio HS-70W, pito Fox 40 y decámetro.

\section{Análisis estadístico}

Para indicar con precisión el perfil de los participantes del programa, fueron determinados el mínimo, máximo, error estándar de la media y la desviación estándar en las variables edad cronológica, altura, peso, estatura media de los padres, predicción de estatura final, edad pico de velocidad de crecimiento, sumatoria de 3 y 7 pliegues, porcentaje graso y porcentaje libre de grasa para el total de la población.

Por otra parte, fueron graficados los resultados de los protocolos de evaluación física para T1, T2 y T3 mediante el programa Graphpad Prism, versión 5.0. Se usó el test de Wilcoxon para comparar las medias de los 
resultados T1 y T3 para cada protocolo de evaluación física, con el programa SPSS, versión 24.0.

\section{Resultados}

La estadística descriptiva del perfil de los participantes se resume en las tablas 2 y 3 . Se evidencia que la edad cronológica de los hombres era de $13.87 \pm 0.94$ años y la de las mujeres, de $14.83 \pm 1.38$ años. En los hombres la predicción de estatura adulta fue de $174.79 \pm 4.09 \mathrm{~cm}$ y una edad pico de velocidad de crecimiento de $14.73 \pm 0.82$ años; en las mujeres la predicción de estatura adulta fue de $165.73 \pm 6.39 \mathrm{~cm}$ y una edad pico de velocidad de crecimiento de $13.25 \pm 0.46$ años. En cuanto al porcentaje de grasa corporal, las mujeres obtuvieron $21.83 \pm 5.64 \%$ y los hombres, $15.46 \pm 5.19 \%$. Según la prueba de maduración biológica, tres de los varones participantes estaban en un nivel de maduración normal y tres en el nivel de maduración atrasada; se obtuvieron los mismos resultados en el grupo de mujeres. Ninguno de los participantes presentó un nivel adelantado; tampoco había alcanzado el estado maduro.

Tabla 2. Estadística descriptiva para hombres $(\mathrm{n}=6)$

\begin{tabular}{|c|c|c|c|c|}
\hline Variable & Unidad & Valor & SEM & $\begin{array}{c}\text { Desviación } \\
\text { estándar }\end{array}$ \\
\hline $\begin{array}{c}\text { Edad } \\
\text { cronológica }\end{array}$ & años & 13.87 & 0.38 & 0.94 \\
\hline Altura & $\mathrm{cm}$ & 161.17 & 2.72 & 6.65 \\
\hline Peso & $\mathrm{kg}$ & 45.82 & 2.27 & 5.57 \\
\hline $\begin{array}{c}\text { Estatura media } \\
\text { padres }\end{array}$ & $\mathrm{cm}$ & 165.67 & 1.80 & 4.40 \\
\hline $\begin{array}{c}\text { Predicción } \\
\text { estatura adulta } \\
\text { (Kamis y Roche, } \\
\text { 1995) }\end{array}$ & $\mathrm{cm}$ & 174.79 & 1.67 & 4.09 \\
\hline $\begin{array}{c}\text { EPVC } \\
\text { (maturity offset, } \\
\text { según Mirwald, } \\
[2002])\end{array}$ & años & 14.73 & 0.34 & 0.82 \\
\hline$\sum 3$ pliegues & mm & 23.17 & 3.67 & 9.00 \\
\hline
\end{tabular}




\begin{tabular}{|c|c|c|c|c|}
\hline Variable & Unidad & Valor & SEM & $\begin{array}{c}\text { Desviación } \\
\text { estándar }\end{array}$ \\
\hline$\sum 7$ pliegues & $\mathrm{mm}$ & 65.00 & 10.24 & 25.09 \\
\hline $\begin{array}{c}\text { \% grasa } \\
\text { (Slaughter } \text { et al. } \\
1988)\end{array}$ & $\%$ & 15.46 & 2.12 & 5.19 \\
\hline$\%$ libre de grasa & $\%$ & 84.55 & 2.12 & 5.19 \\
\hline
\end{tabular}

*EPVC: edad pico velocidad de crecimiento; \% libre de grasa $=(100 \%-\%$ grasa $)$

Fuente: elaboración propia.

Tabla 3. Estadística descriptiva para mujeres $(n=6)$

\begin{tabular}{|c|c|c|c|c|}
\hline Variable & Unidad & Valor & SEM & $\begin{array}{c}\text { Desviación } \\
\text { estándar }\end{array}$ \\
\hline $\begin{array}{c}\text { Edad } \\
\text { cronológica }\end{array}$ & años & 14.83 & 0.57 & 1.38 \\
\hline Altura & $\mathrm{cm}$ & 154.22 & 2.88 & 7.06 \\
\hline Peso & $\mathrm{kg}$ & 49.45 & 3.74 & 9.15 \\
\hline $\begin{array}{c}\text { Estatura media } \\
\text { padres }\end{array}$ & $\mathrm{cm}$ & 163.75 & 1.54 & 3.78 \\
\hline $\begin{array}{c}\text { Predicción } \\
\text { estatura adulta } \\
\text { (Kamis y Roche, } \\
\text { 1995) }\end{array}$ & $\mathrm{cm}$ & 165.73 & 2.61 & 6.39 \\
\hline $\begin{array}{c}\text { EPVC } \\
\text { (maturity offset, } \\
\text { según Mirwald, } \\
[2002])\end{array}$ & años & 13.25 & 0.19 & 0.46 \\
\hline$\sum 3$ pliegues & $\mathrm{mm}$ & 37.00 & 4.97 & 12.18 \\
\hline$\sum 7$ pliegues & $\mathrm{mm}$ & 86.83 & 10.65 & 26.09 \\
\hline $\begin{array}{c}\text { \% grasa } \\
\text { (Slaughter et al., } \\
\text { 1988) }\end{array}$ & $\%$ & 21.83 & 2.30 & 5.64 \\
\hline$\%$ libre de grasa & $\%$ & 78.17 & 2.30 & 5.64 \\
\hline
\end{tabular}

*EPVC: edad pico velocidad de crecimiento; \% libre de grasa $=(100 \%-\%$ grasa $)$

Fuente: elaboración propia. 
Los resultados de las evaluaciones físicas según sexo para salto horizontal, velocidad en $20 \mathrm{~m}$ y agilidad $10 \times 5 \mathrm{~m}$ en T1, T2 y T3 fueron graficadas. Las mejoras encontradas después del programa ejecutado se pueden observar en la tabla 4, que resume los resultados de comparación de medias. En salto horizontal, los hombres pasaron de $173.5 \pm 19.01 \mathrm{~cm}$ a $191.3 \pm 19.85 \mathrm{~cm}$, aumentando $17.8 \mathrm{~cm}$; mientras que las mujeres pasaron de $155 \pm 6.06 \mathrm{~cm}$ a $163.5 \pm 10.13 \mathrm{~cm}$, aumentando $8.5 \mathrm{~cm}$. Estos resultados pueden evidenciarse en la figura 2.

En la prueba de velocidad de $20 \mathrm{~m}$, las mejoras en hombres fueron de $4.17 \pm 0.26 \mathrm{~s}$ a $3.81 \pm 0.22 \mathrm{~s}$ después de la octava semana, mejorando $0.36 \mathrm{~s}$; las mujeres pasaron de $4.37 \pm 0.24 \mathrm{~s}$ a $4.18 \pm 0.15 \mathrm{~s}$, mejorando $0.19 \mathrm{~s}$ después de la octava semana. Estos resultados pueden evidenciarse en la figura 3. La última prueba fue la de agilidad $10 \times 5 \mathrm{~m}$, en donde los hombres de $20.46 \pm 0.4 \mathrm{~s}$ pasaron a $19.65 \pm 0.77 \mathrm{~s}$, mejorando $0.81 \mathrm{~s}$ y las mujeres de $21.01 \pm 0.84 \mathrm{~s}$ a $20.73 \pm 1.06 \mathrm{~s}$, mejorando $0.28 \mathrm{~s}$. Estos resultados se ilustran en la figura 4.

Figura 2. Resultado para salto horizontal en T1, T2 y T3

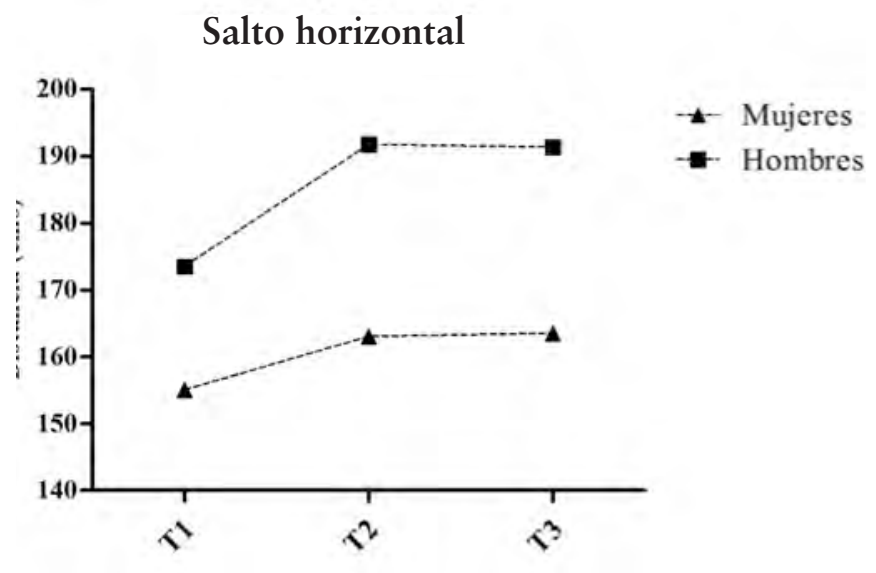

Fuente: elaboración propia. 
Figura 3. Resultado para 20 m en T1 T2 y T3

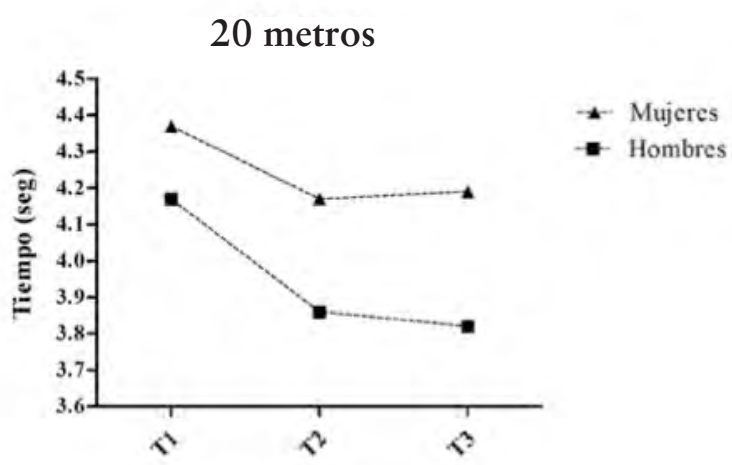

Fuente: elaboración propia.

Figura 4. Resultado para agilidad 10 x 5 m en T1, T2 y T3

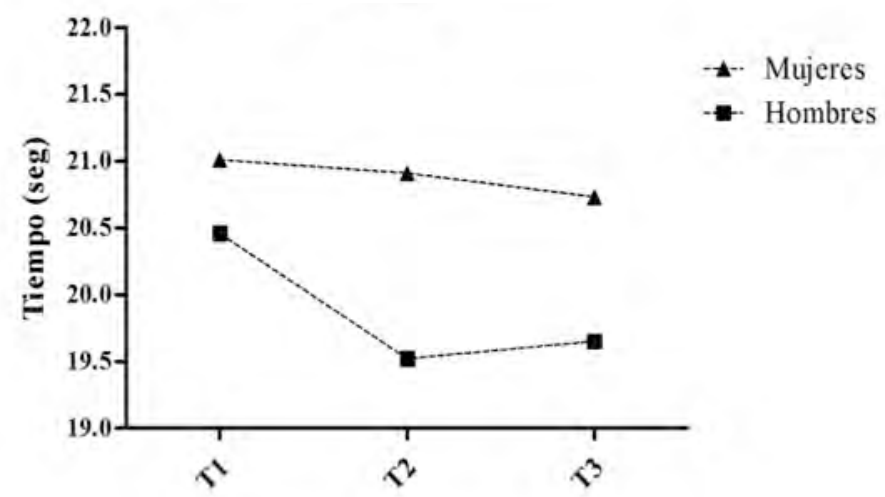

Fuente: elaboración propia. 
Tabla 3. Estadística descriptiva para mujeres $(\mathrm{n}=6)$

\begin{tabular}{|c|c|c|c|c|}
\hline & \multicolumn{2}{|c|}{ Hombres } & \multicolumn{2}{c|}{ Mujeres } \\
\hline Protocolo & $\begin{array}{c}\text { T1 } \\
(\text { media } \pm S D)\end{array}$ & $\begin{array}{c}\text { T3 } \\
(\text { media } \pm S D)\end{array}$ & $\begin{array}{c}\text { T1 } \\
(\text { media } \pm S D)\end{array}$ & $\begin{array}{c}\text { T3 } \\
(\text { media } \pm S D)\end{array}$ \\
\hline Salto $(\mathrm{cm})$ & $173.5 \pm 19.01$ & $191.3 \pm 19.8$ & $155 \pm 6.06$ & $163.5 \pm 10.1^{*}$ \\
\hline Velocidad $20 \mathrm{~m}$ & $4.17 \pm 0.26$ & $3.81 \pm 0,22^{*}$ & $4.37 \pm 0.24$ & $4.18 \pm 0.15$ \\
\hline Agilidad 10 x $5 \mathrm{~m}$ & $20.46 \pm 0.48$ & $19.65 \pm 0.7^{*}$ & $21.01 \pm 0.84$ & $20.73 \pm 1.06$ \\
\hline
\end{tabular}

*p $\leq 0.05$

Fuente: elaboración propia.

El resultado del test de Wilcoxon al comparar T1 y T3 en salto horizontal de hombres fue de 0.058 y de 0.042 para mujeres; para la prueba de velocidad en $20 \mathrm{~m}$, se obtuvo 0.027 para hombres y 0.116 para mujeres, mientras que el resultado del test para la prueba de agilidad $10 \times 5 \mathrm{~m}$ fue de 0.028 para hombres y de 0.600 mujeres. Por lo tanto, se encontró que a pesar de las mejoras en el total de los protocolos físicos aplicados, solo hubo diferencias significativas en velocidad de $20 \mathrm{~m}$ y agilidad 10 × $5 \mathrm{~m}$ para hombres.

\section{Discusión}

Fueron evidentes las mejoras en tiempo y marca de los tenistas élite que participaron en el programa de entrenamiento pliométrico y de velocidad de ocho semanas, sobre los protocolos de velocidad $(20 \mathrm{~m})$, agilidad (10 x 5 m) y fuerza explosiva en miembros inferiores (salto horizontal). En el salto horizontal los hombres aumentaron $17.8 \mathrm{~cm}$, en comparación con la primera prueba, de manera similar a los resultados obtenidos por FernándezFernández et al. (2016), el cual encontró mejoras de $16 \mathrm{~cm}$ en tenistas entre 12 y 13 años, después del mismo periodo de entrenamiento. Por otra parte, y con un programa de cuatro semanas de duración en estudiantes de quinto grado, las mejoras fueron únicamente de $5.6 \mathrm{~cm}$ (Negrea, Negrea y Theodor, 2010). Es importante mencionar que la mayor parte de estudios enfocados en la pliometría para deportistas, utilizan como medio de control protocolos 
como los de salto vertical, agilidad o velocidad (Mateescu, 2013; Váczi et al., 2013; Alptekin et al., 2013; Mohd et al., 2014; Jastrzebski et al., 2014).

Las mejoras en el test de 20 metros fueron de $0.36 \mathrm{~s}$ en los hombres, y $0.19 \mathrm{~s}$ en las mujeres. El tiempo final después del periodo de entrenamiento en las mujeres fue casi el mismo de los hombres en la primera evaluación (T1). Fernández-Fernández et al. (2016) encontraron menores mejoras en esta prueba $(0.13 \mathrm{~s})$, sin embargo, hay que tener en cuenta las diferencias importantes que existen en la calidad de la recolección de información y la tecnología usada.

En cuanto a agilidad, existieron mejoras tanto en hombres como en mujeres, disminuyendo el tiempo inicial en $0.81 \mathrm{~s} \mathrm{y} 0.28 \mathrm{~s}$, respectivamente. En contraste con la prueba utilizada en este estudio, Vaczi et al. (2013) realizaron el entrenamiento pliométrico con una muestra de futbolistas, evaluando la agilidad con el T-test, que también es usado en tenis de campo y el Illinois agility test. Las diferencias encontradas fueron significativas $(\mathrm{p} \leq 0.05)$.

Para Malina et al. (2004) es importante tener en cuenta el estado de maduración y la posible proyección física en el futuro cuando se trabaja con niños y jóvenes, y varios estudios han demostrado que los mejores resultados en protocolos de estado físico los tienen jóvenes que están adelantados maduracionalmente (Malina et al., 2004, 2014; Baxter-Jones et al., 1995). En este estudio solamente se encontraron jóvenes en niveles de maduración normal y atrasada, teniendo en cuenta que la muestra fue muy pequeña.

Este estudio realizó una descripción del perfil antropométrico de los deportistas participantes, en la cual se tuvieron en cuenta algunas variables como peso, altura, porcentaje graso y masa libre de grasa. Al comparar los resultados con los del estudio de Sánchez, Muñoz, Sanz y Zabala (2007), se evidencia cierta similitud en cuanto a composición corporal, a pesar de que en el estudio citado participaron jóvenes tenistas de la élite mundial.

El investigar los efectos del entrenamiento pliométrico y de velocidad en tenistas junior de la Liga de Tenis de Bogotá constituye este trabajo en uno de los pocos existentes en esta área, así como en un aporte importante, no solo teórico-científico, sino también práctico, pues los entrenadores que trabajan en la preparación física pueden tomar en consideración los resultados obtenidos en esta investigación para mejorar la calidad en los resultados y rendimiento de sus deportistas. 
Finalmente, como limitantes de este trabajo, hay que mencionar la falta de herramientas tecnológicas y el tamaño reducido de la muestra, dado que afectaron la calidad de la información y la relevancia en los hallazgos. Igualmente, hay que resaltar la necesidad de un grupo control; sin embargo, los autores reconocen estas limitantes como una oportunidad de mejora para futuros proyectos investigativos.

\section{Conclusión}

Se puede concluir que tenistas junior élite de la Liga de Tenis de Bogotá, entre las edades comprendidas de 12.81-16.38 años, pueden mejorar en pruebas como salto horizontal, velocidad de $20 \mathrm{~m}$ y agilidad de 10 × 5 $\mathrm{m}$ con un programa de ocho semanas de entrenamiento pliométrico y de velocidad, con más influencia en hombres que en mujeres. La transferencia de este trabajo de fuerza al campo de tenis puede ser importante para el aumento de rendimiento, ya que el tenis de campo es un deporte que requiere velocidad de desplazamientos, fuerza explosiva y cambios de dirección constantemente.

\section{Referencias}

Alptekin, A. Kilic, O. y Mavis, M. (2013). The Effect of an 8-week Plyometric Training Program on Sprint and Jumping Performance. Serbian Journal of Sports Sciences, 7(2), 45-50.

Baxter-Jones, A., Helms, P., Maffulli, J., Baines-Preece, J. y Preece M. (1995). Growth and development of male gymnasts, swimmers, soccer and tennis players: a longitudinal study. Annals of Human Biology, 22(5), 381-394.

Berdejo, D. y González, J. M. (2009). Entrenamiento de la fuerza en jóvenes tenistas. Journal of Sport and Health Research, 1(1), 46-55.

Chu, D. Reid, M., Quinn, A. y Crespo, M. (2003). Fuerza y condición física para el tenis. España: ITF.

Fernández-Fernández, J., Kinner, V. y Ferrauti, A. (2010). The Physiological Demands of Hitting and Running in Tennis on Different Surfaces. Journal of Strength and Conditioning Research, 24(12), 3255-3264. 
Fernández-Fernández, J., Saez de Villarreal, E., Sanz Rivas, D. y Moya, M. (2016). The effects of 8-Week Plyometric Training on Physical Performance in Young Tennis Players. Pediatric Exercise Science, (1), 77-86.

Fernández-Fernández, J., Sanz-Rivas, D. y Mendez Villanueva, A. (2009). A Review of the Activity Profile and Physiological Demands of Tennis Match Play. Strength and Conditioning Journal, 31(4), 15-26.

Jastrzebski, Z, Mikolajewski, R., Radziminski, L., Wnorowski, K. y Jaskulska, E. (2014). The effect of a 6-week plyometric training on explosive power in Volleyball players. Baltic Journal of Health and Physical Activity, 6(2), 79-89.

Kovacs, M. S. (2006). Applied physiology of tennis performance. British Journal of Sports Medicine, 40, 381-386.

Kovacs, M. S. (2007). Tennis Physiology Training the Competitive Athlete. Sports Medicine, 37(3), 189-198.

Malina, R. M. (2014). Top 10 Research Questions Related to Growth and Maturation of Relevance to Physical Activity, Performance, and Fitness. Research Quarterly for Exercise and Sport, 85(2), 157-173. doi:10.1080/027 01367.2014.897592

Malina, R. M., Bouchard, C. y Bar, O. (2004). Growth, maturation and physical activity, second edition. Champaign: Human Kinetics.

Mateescu, A. (2013). Plyometrics and the Effect of Four Typical Vertical Height. ARENA-Journal of Physical Activities, (2), 17-29.

Mirwald, R., Baxter, Jones, A. Bailey, D. y Beunen, G. (2002). An assessment of maturity from anthropometric measurements. Medicine \& science in sports \& exercise, 34(4), 689-94. doi:0195-9131/02/3404-0689.

Mohd, M., Syed, A. y Shamsul, M. (2014). The Effectiveness of Plyometric Training on Muscle Strength for Soccer Players. Science, Movement and Health, 14(2), 163-169.

Negrea, V., Negrea, M. y Theodor, D. (2010). Influence of Physical Training Plyometrics Exercises the Fifth Graders. Movement and Health, 10(2), 729-731.

Sánchez-Muñoz, C., Sanz, D. y Zabala, M. (2007). Anthropometric characteristics, body composition and somatotype of elite junior tennis players. British Journal of Sports Medicine, 41(11), 793-799.

Sarabia, J. M., Fernandez Fernandez, J., Juan-Recio, C. Hernández, Davó, H. Urban, T. y Moya, M. (2015). Mechanical, Hormonal and Physiological Effects of a Non-Failure Short-Term Strength Training Program in Young Tennis Players. Journal of Human Kinetics, 45, 81-91. 
Shelvam, P. V. Singh, J. y Singh, G. (2013). Effects of Six Week Plyometric Training and Aerobic Training on Selected Motor Fitness Components Among Basketball Players. International Journal of Sport Sciences and Fitness, 3(1), 117-123.

Slaughter, M. et al. (1988). Skinfold equation for estimation of body fatness in children and youth. Human Biology, 60, 709-23.

Torres-Luque, G., Cabello-Manrique, D., Hernandez-Garcia, R. y Garatachea, N. (2011). An analysis of competition in Young tennis players. European Journal of Sport Science, 11(1), 39-43.

Váczi, M. Tollár, J. Meszler, B., Juhász, I. y Karsai, I. (2013). Short-Term High Intensity Plyometric Training Program Improves Strength, Power and Agility in Male Soccer Players. Journal of Human Kinetics, 36, 17-26. 